\title{
Injury rates and injury risk factors among Federal Bureau of Investigation new agent trainees
}

Joseph J Knapik ${ }^{1 *}$, Tyson Grier ${ }^{1}$, Anita Spiess ${ }^{1}$, David I Swedler ${ }^{2}$ Keith G Hauret ${ }^{1}$, Bria Graham ${ }^{1}$, James Yoder ${ }^{3}$ and Bruce $\mathrm{H}$ Jones ${ }^{1}$

\begin{abstract}
Background: A one-year prospective examination of injury rates and injury risk factors was conducted in Federal Bureau of Investigation (FBI) new agent training.

Methods: Injury incidents were obtained from medical records and injury compensation forms. Potential injury risk factors were acquired from a lifestyle questionnaire and existing data at the FBI Academy.

Results: A total of 426 men and 105 women participated in the project. Thirty-five percent of men and $42 \%$ of women experienced one or more injuries during training. The injury incidence rate was 2.5 and 3.2 injuries/1,000 person-days for men and women, respectively (risk ratio (women/men) $=1.3,95 \%$ confidence interval $=0.9-1.7$ ). The activities most commonly associated with injuries (\% of total) were defensive tactics training (58\%), physical fitness training (20\%), physical fitness testing (5\%), and firearms training (3\%). Among the men, higher injury risk was associated with older age, slower 300-meter sprint time, slower 1.5-mile run time, lower total points on the physical fitness test (PFT), lower self-rated physical activity, lower frequency of aerobic exercise, a prior upper or lower limb injury, and prior foot or knee pain that limited activity. Among the women higher injury risk was associated with slower 300-meter sprint time, slower 1.5-mile run time, lower total points on the PFT, and prior back pain that limited activity.
\end{abstract}

Conclusion: The results of this investigation supported those of a previous retrospective investigation emphasizing that lower fitness and self-reported pain limiting activity were associated with higher injury risk among FBI new agents.

Keywords: Physical training, Age, Body mass index, Physical activity, Physical fitness

\section{Background}

To accomplish its law enforcement and protective missions, the Federal Bureau of Investigation (FBI) trains about 700 new agents each year. New agent training classes at the FBI Academy in Quantico, Virginia, are currently 21-weeks in length. Classes contain a maximum of 50 individuals and new classes begin about every two weeks. During training, new agents are required to participate in a variety of activities which include defensive tactics, practical applications, firearms training, exercise, and classroom/computer activities. Defensive tactics training includes boxing, self-defense,

\footnotetext{
* Correspondence: joseph.knapik@us.army.mil

1 U.S. Army Institute of Public Health, Aberdeen Proving Ground, Maryland, USA

Full list of author information is available at the end of the article
}

and suspect apprehension techniques. Practical application training consists of driver training, conducting surveillance, room entries, room clearing, and arrest procedures. Firearms' training involves marksmanship, close combat skills, and moving in teams with weapons. To graduate, new agents must pass a Physical Fitness Test (PFT) (sit-ups, push-ups, 300-meter sprint, and 1.5-mile run) and tests are routinely administered at least three times during the new agent training course. While at the FBI Academy, most new agents are expected to perform physical fitness training on their own, although those who failed the first PFT are required to attend group physical training three times per week. In addition to physical tasks, FBI new agents spend considerable time in the classroom involved in lectures and on-line training on law enforcement topics 
and investigative techniques. The curriculum currently has over 900 hours of training, including 94 hours of defensive tactics training, 95 hours of practical application training, and 114 hours of firearms training.

The physical activities performed by new agents place them at risk of injury. We previously reported on a retrospective investigation of injury rates, physical fitness and the association of these in FBI new agent training [1]. The purpose of the present investigation was to expand on this earlier project by prospectively examining a larger number of potential risk factors in a separate cohort of FBI new agents.

\section{Methods}

This investigation employed a prospective cohort design. On the second day of the FBI new agent training course, individuals completed a PFT. Early on the third day, new agents were briefed on the project and those who consented to participate signed a form and completed a questionnaire. The questionnaire contained items on tobacco use, prior physical activity, prior pain and injuries, menstrual history, date of birth, height, and weight. Injuries during training were tracked from a database at the FBI Academy Health Clinic, as described below. The project took place over the course of a year (March 2009-March 2010) with new agents enrolled in the investigation as new classes started. Participants were followed until they graduated or attrited from training. The FBI's Human Use Review Committee approved the investigation.

\section{Physical fitness data}

PFT scores were obtained from the Physical Training Unit (PTU) of the FBI Academy. The PTU administered the PFT using well-standardized methods. The PFT events were identical for men and women and consisted of four scored events administered in this order: pushups requiring continuous motion to exhaustion, 1-minute bent-leg sit-ups, a 300-meter sprint, and a 1.5-mile run. Pull-ups to exhaustion were also tested as the last event, but this event was not considered in the total PFT score. At least 5 minutes of rest were provided between events. Gender-specific points were assigned to various levels of performance on each PFT event. To pass the PFT, a point score of 12 was required, with at least 1 point achieved on each event (maximum possible score was 40 points). Details of the PFT events and scoring system are provided on-line [2].

\section{Final student status}

Data on final student status were obtained from a list provided by the Training Director's Office at the Academy. This indicated students who did not graduate, the date they left the course, and the reasons for attrition.

\section{Injury data}

Medical care providers at the FBI Academy Health Clinic at Quantico, Virginia, routinely entered information on medical encounters into a computerized database. Trained personnel examined each new agent medical encounter and determined whether it was for an injury (defined below) or for other medical care. For each injury encounter, information extracted included the date of the visit, the type of visit (new injury visit or follow-up on a previous visit), diagnosis, anatomical location, and activity associated with the injury. In addition to the encounters in the medical database, injury information was also obtained from the United States Department of Labor's CA-1 forms (Federal Employee's Notice of Traumatic Injury and Claim for Continuation of Pay/Compensation). This form provided additional information on the diagnosis, anatomical location, and the activity associated with the injury.

An injury case was defined as a new agent who sustained physical damage to the body [3] and sought medical care or medical compensation one or more times during the FBI new agent training course. Injuries were grouped by "type" as determined from descriptive information in the medical notes and/or CA-1, and by the specific diagnosis. Injury types included 1) overuse injury, 2) traumatic injury, 3) environmental/other injury, and 4) any injury. Overuse injuries were those due to, or related to, long-term repetitive energy exchanges, resulting in cumulative microtrauma. Specific overuse injury diagnoses included tendonitis, bursitis, fasciitis, muscle injury associated with overuse (strain), joint injury associated with overuse (sprain), retropatellar pain syndrome, impingement, degenerative joint conditions, shin splints and musculoskeletal pain (not otherwise specified). Traumatic injuries were those due to sudden energy exchanges (acute event), resulting in abrupt overload with tissue trauma. Specific traumatic injury diagnoses included muscle injury associated with an acute event (strain), joint injury associated with an acute event (sprain), dislocation, fracture, abrasion, laceration, contusions, closed head injury/concussion and pain (associated with an acute event). Environmental/ other injuries were those due to exposure to weather, animals, or chemicals and included heat-related injuries, insect bites, chemical exposures, and exertion-related events. "Any injury" included overuse and trauma diagnoses as described above, but excluded environmental/ other injuries. The "any injury" type included primarily musculoskeletal injuries, but also included dermatological events (e.g., abrasions, lacerations).

New injuries were first patient encounters resulting in a particular injury diagnosis at a particular anatomical location. Follow-up injuries were subsequent encounters for the same injury. If follow-up visits occurred, they were used in conjunction with the initial encounter to 
determine the final diagnosis for a specific injury. Thus an initial diagnosis could be changed as a result of a more specific diagnosis at a higher level of medical care.

\section{Data analysis}

All analyses were performed with Predictive Analytic Software, Version 18.0.0. Cumulative injury incidences for any injury, overuse injury, and traumatic injury were calculated as:

(new agents with $\geq 1$ injury $\div$ total number of new agents) $\times 100 \%$

Injury incidence rates for any injury, overuse injury, and traumatic injury were calculated as:

(new agents with $\geq 1$ injury $\div$ total number of new training) $\times 100 \%$

Chi-square statistics were used to make comparisons among men and women on injury incidence; a chisquare for person time was used to compare men and women on injury incidence rates.

Cox regression (survival analysis) was used to examine the association between the time to the first injury (any injury) and independent variables (potential injury risk factors) from the PFT and questionnaire. For each analysis, once a new agent had an injury, his or her contribution to time in training was terminated (censored). Those who attrited from training had their time censored at the day they left training, unless their time had already been censored as the result of an injury. All potential risk factors were entered into the regression models as categorical variables. Continuous variables were converted to quartiles (four groups of approximately equal size) or tertiles (three groups of approximately equal size) based on the distribution of scores. Body mass index (BMI) was also examined in categories according to National Institute of Health Guidelines [4]. Some categories of nominal and ordinal variables were combined to increase statistical power. Because of the relatively narrow age range (24-39 years), age was categorized into only two groups, above and below 30 years of age. For all Cox regressions, simple contrasts were used, comparing the hazard at a baseline level of a variable (defined with a hazard ratio (HR) of 1.00 and called the "referent") with other levels (strata) of the same variable. Univariate Cox regressions established the individual associations between time to first injury at each stratum of a variable. Variables were included in a multivariate backward stepping Cox regression if they achieved $p<0.10$ in the univariate analyses [5]. Multivariate Cox regressions established the effect of multiple independent risk factors on injury risk.

\section{Results}

There were a total of 531 new agents who enrolled in the project, 426 men and 105 women. Of the 534 new agents who were briefed, three declined to participate. Of the 531 who enrolled, $12 \%(n=50)$ of men and $33 \%$ $(n=35)$ of women did not complete the FBI new agent course. Among the men, injuries and failure to pass the PFT accounted for $26 \%(n=13)$ and $14 \%(n=7)$ of the attrition cases, respectively. Among the women, injuries and failure to pass the PFT accounted for $14 \%(\mathrm{n}=5)$ and $3 \%(n=1)$ of the attrition cases, respectively.

\section{Descriptive data on injuries}

Table 1 shows the number and proportion of new injuries and follow-ups by diagnosis. Overuse injuries made up $14 \%$ of the new injury cases while traumatic injuries made up $68 \%$. Diagnoses with the largest number of new injury cases were traumatic sprains (joint injuries), traumatic strains (muscle injuries), musculoskeletal pain associated with trauma, abrasions/lacerations, contusions, and insect bites. These six diagnoses accounted for $70 \%$ of all new injury cases. Musculoskeletal pain involved medical encounters in which an individual reported pain in a specific musculoskeletal location but no specific diagnosis was provided in the record. The determination that this was a traumatic- or overuse-type injury was based on notes indicating that the complaint was associated with a sudden or acute event (traumatic) or that the pain had occurred gradually over time (overuse).

The most common anatomical locations for new injuries were the knees $(9.8 \%)$, thighs $(7.7 \%)$, shoulders (7.6\%), fingers $(7.3 \%)$, lower back $(5.7 \%)$, face $(5.7 \%)$, head (5.1\%), and ankles (4.8\%). For follow-ups, the most common anatomical locations were the ankle (20.5\%), shoulder (13.6\%), knees (9.1\%), thigh $(9.1 \%)$, and head (9.1\%).

Table 2 shows new injuries and follow-ups by the training activity associated with the injury. Two activities, defensive tactics and physical fitness training, were associated with $78 \%$ of the new injury cases and $86 \%$ of the follow-up cases.

\section{Cumulative injury incidence and injury rates}

Table 3 shows cumulative injury incidence and injury incidence rate by gender and injury type. Overall injury incidence (any injury) was slightly higher among the women, the result of a higher incidence of both overuse and traumatic injuries. In consonance with the injury incidence, the overall injury incidence rate (any injury) was slightly higher among the women, accounted for by a higher incidence of both overuse and traumatic injuries. The men had a mean \pm standard deviation (SD) of $137 \pm 27$ days in training while the women had $130 \pm$ 29 days. The total days of training for men were 58,550 and for the women 13,680 . 
Table 1 Injury cases by diagnoses

\begin{tabular}{|c|c|c|c|c|c|c|}
\hline \multirow[t]{2}{*}{ Type of Injury } & & \multirow[t]{2}{*}{ Diagnosis } & \multicolumn{2}{|c|}{ New Injuries } & \multicolumn{2}{|c|}{ Follow-Ups } \\
\hline & & & $N$ & $\%$ & $\mathrm{~N}$ & $\%$ \\
\hline \multirow[t]{18}{*}{ ANY INJURY } & OVERUSE & Tendonitis & 10 & 3.2 & 3 & 6.8 \\
\hline & & Bursitis & 2 & 0.6 & 0 & 0.0 \\
\hline & & Retropatellar pain syndrome & 5 & 1.6 & 2 & 4.5 \\
\hline & & Muscle injury (overuse strain) & 14 & 4.4 & 2 & 4.5 \\
\hline & & $\underline{\text { Neurological }}$ & 1 & 0.3 & 0 & 0.0 \\
\hline & & Joint injury (overuse sprain) & 4 & 1.3 & 1 & 2.3 \\
\hline & & Musculoskeletal pain (overuse) & 6 & 1.9 & 0 & 0.0 \\
\hline & & Shin splints & 1 & 0.3 & 0 & 0.0 \\
\hline & TRAUMATIC & Muscle injury (traumatic strain) & 39 & 12.4 & 5 & 11.4 \\
\hline & & Joint injury (traumatic sprain) & 45 & 14.3 & 13 & 29.5 \\
\hline & & Musculoskeletal pain (traumatic) & 37 & 11.7 & 3 & 6.8 \\
\hline & & Dislocation & 7 & 2.2 & 0 & 0.0 \\
\hline & & Bone Fracture & 4 & 1.3 & 2 & 4.5 \\
\hline & & Tooth Fracture & 2 & 0.6 & 0 & 0.0 \\
\hline & & Nasal Fracture & 2 & 0.6 & 0 & 0.0 \\
\hline & & Abrasion or laceration & 33 & 10.5 & 2 & 4.5 \\
\hline & & Contusion & 33 & 10.5 & 4 & 9.1 \\
\hline & & Closed Head Injury/Concussion & 11 & 3.5 & 4 & 9.1 \\
\hline \multirow[t]{5}{*}{ ENVIR/OTHER } & & General heat-related injury & 3 & 1.0 & 2 & 4.5 \\
\hline & & Exertion & 11 & 3.5 & 0 & 0.0 \\
\hline & & Insect bites or stings & 33 & 10.5 & 1 & 2.3 \\
\hline & & Chemical Burn (capsicum spray) & 12 & 3.8 & 0 & 0.0 \\
\hline & Total Cases & & 315 & 100.0 & 44 & 100.0 \\
\hline
\end{tabular}

\section{Univariate analysis of injury risk factors}

Table 4 shows the univariate associations between any injury and age, physical characteristics, and physical fitness. Most women (74\%) could not perform pull-ups, so this variable was separated into women who could perform one or more pull-ups and those who could not.

Table 2 New injury cases by associated training activity

\begin{tabular}{lcccc}
\hline Activity & \multicolumn{2}{c}{ New Injuries } & \multicolumn{2}{c}{ Follow-Ups } \\
\cline { 2 - 5 } & $\mathbf{N}$ & $\mathbf{\%}$ & $\mathbf{N}$ & $\%$ \\
\hline Defensive Tactics & 184 & 58.4 & 25 & 56.8 \\
\hline Physical Fitness Training & 63 & 20.0 & 13 & 29.5 \\
\hline Physical Fitness Testing & 15 & 4.8 & 2 & 4.5 \\
\hline Firearms Training & 8 & 2.5 & 0 & 0.0 \\
\hline Off-Duty, Academy & 1 & 0.3 & 0 & 0.0 \\
\hline Off Duty, Not Academy & 6 & 1.9 & 0 & 0.0 \\
\hline Practical Applications Training & 5 & 1.6 & 1 & 2.3 \\
\hline Sports & 1 & 0.3 & 0 & 0.0 \\
\hline Other & 6 & 1.9 & 0 & 0.0 \\
\hline Unknown & 26 & 8.2 & 3 & 6.8 \\
\hline Total & 315 & 100.0 & 44 & 100.0 \\
\hline
\end{tabular}

There was little association between injury risk and physical characteristics, although men in the third highest quartile of BMI had higher injury risk than those in the first quartile. Among the men, higher injury incidence was associated with older age, slower 300-meter sprint time, slower 1.5-mile run time, and fewer total PFT points. Among the women, higher injury incidence was associated with slower 300-meter sprint time, slower 1.5-mile run time, and fewer total PFT points. Injury risk tended to be elevated for both men and women in the lower quartiles of performance for all physical fitness measures.

Table 5 shows the univariate associations between any injury and the questionnaire variables. Among the men, higher injury risk was associated with having never smoked, a lower self-rating of physical activity, and a lower frequency of aerobic exercise in the past 2 months. Men who reported a prior upper or lower limb injury were at higher injury risk. If the men reported that a limb injury prevented physical activity for at least 1 week, or that they had not returned to normal activity since the injury, the risk of injury was further elevated. Men who reported having foot or knee pain that limited 
Table 3 Cumulative injury incidence and injury incidence rates by gender and injury type

\begin{tabular}{|c|c|c|c|c|c|}
\hline & Injury Type & Men & Women & Risk Ratio - Women/Men (95\% Confidence Interval) & p-value ${ }^{a}$ \\
\hline \multirow[t]{3}{*}{ Cumulative Injury Incidence (\%) } & Any & 35.0 & 41.9 & $1.20(0.93-1.55)$ & 0.19 \\
\hline & Overuse & 5.6 & 13.3 & $2.36(1.27-4.42)$ & $<0.01$ \\
\hline & Traumatic & 31.2 & 37.1 & $1.19(0.90-1.58)$ & 0.25 \\
\hline \multirow[t]{3}{*}{ Injury Incidence Rate (injuries/1,000 person-days) } & Any & 2.54 & 3.22 & $1.26(0.90-1.77)$ & 0.09 \\
\hline & Overuse & 0.41 & 1.02 & $2.50(1.29-4.83)$ & $<0.01$ \\
\hline & Traumatic & 2.27 & 2.85 & $1.26(0.88-1.79)$ & 0.11 \\
\hline
\end{tabular}

${ }^{\mathrm{a} C h i-s q u a r e ~ s t a t i s t i c}$

activity were at elevated injury risk; back pain limiting activity also elevated injury risk but less so than foot or knee pain. Few of the questionnaire variables were significantly associated with injury among the women. Back pain that limited activity was associated with elevated injury risk.

\section{Multivariate analysis of injury risk factors}

Table 6 shows the results of the multivariate backwardstepping Cox regression examining associations between any injury and the risk factors. Among the men, independent risk factors for injuries included older age, slower sprint speed, slower 1.5 -mile run time, having not smoked 100 cigarettes in their lifetime, lower self-rating of physical activity, lower and higher frequency of aerobic training, having a prior upper limb injury (especially if that injury did not allow return to normal physical activity), and having knee pain that limited physical activity. Among the women, independent risk factors for injuries included slower 1.5-mile run time and back pain that limited physical activity.

\section{Discussion and conclusion}

The project reported here complemented an earlier retrospective investigation [1] of injuries and physical fitness among FBI new agents by prospectively examining injury rates and exploring a greater number of potential injury risk factors. In the previous retrospective investigation [1], the only risk factors for injury examined were physical fitness measures. In the present prospective investigation, the association between injuries and low physical fitness was replicated, and additional potential risk factors were examined including age, physical characteristics, tobacco use, prior physical activity, self-assessed fitness, prior injury, and menstrual history.

The earlier retrospective investigation [1] reported an overall injury incidence (any injury) of $40 \%$ for men and $45 \%$ for women in the years 2003 through 2008 . The present investigation found a slightly lower incidence of injury: $35 \%$ for men and $42 \%$ for women. More specific injury diagnoses were reported in the present compared to the previous investigation [1]. For example, in the retrospective investigation, traumatic musculoskeletal pain (not otherwise specified) accounted for $27 \%$ of the diagnoses while in the present investigation only $12 \%$ of the diagnoses were in this category. The higher diagnostic specificity in the present investigation was likely due to the presence of a full-time physician who was on site at the FBI Health Clinic for the entire period and who made many of the diagnoses. In the retrospective investigation, a physician was on-site for only short periods and on-site nurses made most of the diagnoses.

The specific diagnoses (i.e., exclusive of musculoskeletal pain) that comprised the largest number of new injury cases were strains, sprains, contusions, and abrasions/ lacerations. These are common injuries in physically active groups of individuals who are involved in running, sports, recreational activities, and military training [6-16]. Less serious traumatic injuries such as abrasions/lacerations and contusions each accounted for $11 \%$ of all injuries. This is comparable to the sports literature in which abrasions and lacerations have accounted for $8 \%-11 \%$ of all injuries $[9,12,14]$, and contusions $6 \%-24 \%[8,11,13,14]$. With regard to overuse injuries, tendonitis accounted for less than 2\%-3\% of FBI new agent injuries, but in runners, college athletes, and military trainees this injury accounts for $5 \%-12 \%$ [6-8,10,12,15]. There were few cases of more serious traumatic injuries such as fractures, dislocations, and subluxations; these totaled only $4 \%$ of all injuries. In studies of runners and collegiate sports athletes, fractures, subluxations, and dislocations have accounted for 3\%-13\% of all injuries [6,8,9,11-14]. It appears that more serious injuries were less common in FBI new agent training than in many other groups of active individuals.

From an injury-prevention standpoint, the most important information in the medical records was the activity associated with the injury. In some cases, the injury was of the overuse type and had an insidious onset making it difficult to link to a specific event. In most cases, the injury could be linked to a specific training activity, but recording of activities associated with injury was not standardized in the medical records so that pinpointing the injury producing event was difficult. For example, although $58 \%$ of the injuries were associated with defensive tactics, often the medical records provided no further information that could have isolated a more specific 
Table 4 Univariate associations between injury risk ${ }^{a}$ and age, physical characteristics, and physical fitness

\begin{tabular}{|c|c|c|c|c|c|c|c|c|c|c|}
\hline \multirow[t]{2}{*}{ Variable } & \multicolumn{5}{|l|}{ Men } & \multicolumn{5}{|l|}{ Women } \\
\hline & Strata & $\mathbf{N}$ & $\begin{array}{l}\text { Injured } \\
(\%)\end{array}$ & $\begin{array}{l}\text { Hazard Ratios }(95 \% \mathrm{Cl}) \text { from Cox } \\
\text { Regressions }\end{array}$ & $\begin{array}{l}\text { p-value(from Wald } \\
\text { statistic) }\end{array}$ & Strata & $\mathrm{N}$ & $\begin{array}{l}\text { Injured } \\
(\%)\end{array}$ & $\begin{array}{l}\text { Hazard Ratios }(95 \% \mathrm{Cl}) \text { fromCox } \\
\text { Regressions }\end{array}$ & $\begin{array}{l}\text { p-value(from } \\
\text { Waldstatistic) }\end{array}$ \\
\hline \multirow[t]{2}{*}{ Age } & $\begin{array}{l}24.4-29.9 \\
\text { Years }\end{array}$ & 188 & 26.6 & 1.00 & Referent & $\begin{array}{l}24.1-29.9 \\
\text { years }\end{array}$ & 60 & 36.7 & 1.00 & Referent \\
\hline & $\begin{array}{l}30.0-38.6 \\
\text { Years }\end{array}$ & 238 & 41.6 & $1.83(1.30-2.57)$ & $<0.01$ & $\begin{array}{l}30.0-37.0 \\
\text { years }\end{array}$ & 45 & 48.9 & $1.52(0.84-2.75)$ & 0.16 \\
\hline \multirow[t]{4}{*}{ Height } & $144-175 \mathrm{~cm}$ & 123 & 35.0 & $1.02(0.69-1.51)$ & 0.93 & $147-162 \mathrm{~cm}$ & 39 & 46.2 & $1.49(0.72-3.10)$ & 0.28 \\
\hline & $176-178 \mathrm{~cm}$ & 73 & 30.1 & $0.79(0.48-1.28)$ & 0.34 & $163-168 \mathrm{~cm}$ & 31 & 45.2 & $1.45(0.67-3.14)$ & 0.35 \\
\hline & $179-180 \mathrm{~cm}$ & 65 & 38.5 & $1.10(0.69-1.75)$ & 0.70 & $169-180 \mathrm{~cm}$ & 35 & 34.3 & 1.00 & Referent \\
\hline & $181-205 \mathrm{~cm}$ & 165 & 35.8 & 1.00 & Referent & & & & & \\
\hline \multirow[t]{4}{*}{ Body Mass } & $51.8-75.9 \mathrm{~kg}$ & 109 & 33.9 & 1.00 & Referent & $47.7-56.8 \mathrm{~kg}$ & 35 & 42.9 & 1.00 & Referent \\
\hline & $76.0-81.8 \mathrm{~kg}$ & 127 & 33.1 & $0.98(0.61-1.57)$ & 0.91 & $56.9-63.6 \mathrm{~kg}$ & 35 & 45.7 & $1.15(0.57-2.33)$ & 0.70 \\
\hline & $81.9-88.6 \mathrm{~kg}$ & 99 & 32.3 & $0.98(0.61-1.57)$ & 0.92 & $63.7-90.9 \mathrm{~kg}$ & 35 & 37.1 & $0.91(0.43-1.91)$ & 0.80 \\
\hline & $88.7-113.6 \mathrm{~kg}$ & 91 & 41.8 & $1.24(0.79-1.95)$ & 0.35 & & & & & \\
\hline \multirow[t]{4}{*}{ Body Mass Index } & $\begin{array}{l}16.51-23.74 \\
\mathrm{~kg} / \mathrm{m}^{2}\end{array}$ & 108 & 34.3 & 1.00 & Referent & $\begin{array}{l}18.25-21.26 \\
\mathrm{~kg} / \mathrm{m}^{2}\end{array}$ & 37 & 43.2 & 1.00 & Referent \\
\hline & $\begin{array}{l}23.75-25.11 \\
\mathrm{~kg} / \mathrm{m}^{2}\end{array}$ & 110 & 25.5 & $0.74(0.46-1.22)$ & 0.24 & $\begin{array}{l}21.27-23.30 \\
\mathrm{~kg} / \mathrm{m}^{2}\end{array}$ & 34 & 44.1 & $1.13(0.56-2.29)$ & 0.73 \\
\hline & $\begin{array}{l}25.12-26.63 \\
\mathrm{~kg} / \mathrm{m}^{2}\end{array}$ & 103 & 48.5 & $1.54(1.00-2.36)$ & 0.05 & $\begin{array}{l}23.31-29.54 \\
\mathrm{~kg} / \mathrm{m}^{2}\end{array}$ & 34 & 38.2 & $0.93(0.45-1.94)$ & 0.85 \\
\hline & $\begin{array}{l}26.64-38.74 \\
\mathrm{~kg} / \mathrm{m}^{2}\end{array}$ & 105 & 32.4 & $0.95(0.60-1.52)$ & 0.84 & & & & & \\
\hline \multirow[t]{3}{*}{ Body Mass Index } & $\begin{array}{l}\begin{array}{l}16.5-24.99 \\
\mathrm{~kg} / \mathrm{m}^{2}\end{array} \\
\end{array}$ & 191 & 30.4 & 1.00 & Referent & $\begin{array}{l}16.5-24.99 \\
\mathrm{~kg} / \mathrm{m}^{2}\end{array}$ & 94 & 42.6 & 1.00 & Referent \\
\hline & $\begin{array}{l}25.00-29.99 \\
\mathrm{~kg} / \mathrm{m}^{2}\end{array}$ & 223 & 36.3 & $1.18(0.89-1.92)$ & 0.26 & $\begin{array}{l}25.00-29.99 \\
\mathrm{~kg} / \mathrm{m}^{2}\end{array}$ & 11 & 36.4 & $0.92(0.33-2.57)$ & 0.87 \\
\hline & $\geq 30 \mathrm{~kg} / \mathrm{m}^{2}$ & 12 & 8.3 & $0.24(0.03-1.72)$ & 0.16 & $\geq 30 \mathrm{~kg} / \mathrm{m}^{2}$ & 0 & -— & -- & -—- \\
\hline \multirow[t]{4}{*}{ Push-Ups } & $\begin{array}{l}1-31 \\
\text { repetitions }\end{array}$ & 104 & 37.5 & $1.20(0.76-1.91)$ & 0.44 & $\begin{array}{l}0-13 \\
\text { repetitions }\end{array}$ & 36 & 50.0 & $1.38(0.67-2.81)$ & 0.38 \\
\hline & $\begin{array}{l}32-36 \\
\text { repetitions }\end{array}$ & 112 & 40.2 & $1.38(0.88-2.16)$ & 0.16 & $\begin{array}{l}14-20 \\
\text { repetitions }\end{array}$ & 35 & 37.1 & $0.95(0.44-2.04)$ & 0.89 \\
\hline & $\begin{array}{l}37-42 \\
\text { repetitions }\end{array}$ & 106 & 30.2 & $0.97(0.60-1.58)$ & 0.91 & $\begin{array}{l}21-39 \\
\text { repetitions }\end{array}$ & 34 & 38.2 & 1.00 & Referent \\
\hline & $\begin{array}{l}43-71 \\
\text { repetitions }\end{array}$ & 104 & 31.7 & 1.00 & Referent & & & & & \\
\hline \multirow[t]{2}{*}{ Sit-Ups } & $\begin{array}{l}28-40 \\
\text { repetition }\end{array}$ & 97 & 42.7 & $1.32(0.83-2.09)$ & 0.23 & $\begin{array}{l}26-40 \\
\text { repetition }\end{array}$ & 38 & 42.1 & $1.28(0.61-2.71)$ & 0.52 \\
\hline & $\begin{array}{l}41-44 \\
\text { repetitions }\end{array}$ & 132 & 34.0 & $1.05(0.65-1.68)$ & 0.84 & $\begin{array}{l}41-44 \\
\text { repetitions }\end{array}$ & 34 & 47.1 & $1.44(0.68-3.05)$ & 0.34 \\
\hline
\end{tabular}


Table 4 Univariate associations between injury risk ${ }^{a}$ and age, physical characteristics, and physical fitness (Continued)

\begin{tabular}{|c|c|c|c|c|c|c|c|c|c|c|}
\hline & $\begin{array}{l}45-47 \\
\text { repetitions }\end{array}$ & 101 & 29.5 & $0.84(0.53-1.34)$ & 0.46 & $\begin{array}{l}45-51 \\
\text { repetitions }\end{array}$ & 33 & 36.4 & 1.00 & Referent \\
\hline & $\begin{array}{l}48-59 \\
\text { repetitions }\end{array}$ & 96 & 35.6 & 1.00 & Referent & & & & & \\
\hline \multirow[t]{4}{*}{ 300-Meter Sprint } & $\begin{array}{l}40-44 \\
\text { seconds }\end{array}$ & 123 & 28.5 & 1.00 & Referent & $\begin{array}{l}46-55 \\
\text { seconds }\end{array}$ & 42 & 35.7 & 1.00 & Referent \\
\hline & $\begin{array}{l}45-46 \\
\text { seconds }\end{array}$ & 132 & 34.1 & $1.23(0.79-1.91)$ & 0.36 & $\begin{array}{l}56-58 \\
\text { seconds }\end{array}$ & 41 & 39.0 & $1.17(0.58-2.37)$ & 0.66 \\
\hline & $\begin{array}{l}47-48 \\
\text { seconds }\end{array}$ & 108 & 39.8 & $1.48(0.95-2.32)$ & 0.08 & $\begin{array}{l}59-62 \\
\text { seconds }\end{array}$ & 22 & 59.1 & $2.23(1.06-4.70)$ & 0.04 \\
\hline & $\begin{array}{l}49-55 \\
\text { seconds }\end{array}$ & 63 & 41.3 & $1.71(1.03-2.84)$ & 0.04 & & & & & \\
\hline \multirow[t]{4}{*}{ 1.5-Mile Run } & $\begin{array}{l}8.18-10.35 \\
\min \end{array}$ & 108 & 25.9 & 1.00 & Referent & $\begin{array}{l}\text { 10.05-12.06 } \\
\min \end{array}$ & 35 & 40.0 & 1.00 & Referent \\
\hline & $\begin{array}{l}\text { 10.36-11.10 } \\
\min \end{array}$ & 107 & 30.8 & $1.24(0.75-2.05)$ & 0.41 & $\begin{array}{l}12.07-12.95 \\
\min \end{array}$ & 35 & 37.1 & $0.90(0.42-1.92)$ & 0.80 \\
\hline & $\begin{array}{l}11.11-11.64 \\
\min \end{array}$ & 104 & 37.5 & $1.60(0.99-2.61)$ & 0.06 & $\begin{array}{l}12.96-14.92 \\
\min \end{array}$ & 35 & 62.9 & $1.95(1.00-3.80)$ & 0.05 \\
\hline & $\begin{array}{l}11.65-15.02 \\
\min \end{array}$ & 106 & 45.3 & $2.06(1.30-3.29)$ & $<0.01$ & & & & & \\
\hline \multirow{4}{*}{$\begin{array}{l}\text { PhysicalFitnessTest } \\
\text { Score }\end{array}$} & 1-11 points & 114 & 43.0 & $1.73(1.09-2.75)$ & 0.02 & 2-10 points & 38 & 47.4 & $2.04(1.00-4.14)$ & 0.05 \\
\hline & $12-14$ points & 107 & 30.8 & $1.15(0.70-1.91)$ & 0.58 & 11-14 points & 32 & 43.8 & $1.77(0.84-3.71)$ & 0.13 \\
\hline & $15-18$ points & 108 & 36.1 & $1.35(0.82-2.17)$ & 0.24 & $15-27$ points & 35 & 34.3 & 1.00 & Referent \\
\hline & 19-37 points & 97 & 28.9 & 1.00 & Referent & & & & & \\
\hline \multirow[t]{4}{*}{ Pull-Ups } & $\begin{array}{l}0-4 \\
\text { repetitions }\end{array}$ & 94 & 41.5 & $1.12(0.72-1.75)$ & 0.62 & 0 repetitions & 78 & 43.6 & $1.17(0.58-2.37)$ & 0.66 \\
\hline & $\begin{array}{l}5-8 \\
\text { repetitions }\end{array}$ & 138 & 33.3 & $0.85(0.55-1.31)$ & 0.46 & $\begin{array}{l}1-12 \\
\text { repetitions }\end{array}$ & 27 & 37.0 & 1.00 & Referent \\
\hline & $\begin{array}{l}\text { 9-11 } \\
\text { repetitions }\end{array}$ & 92 & 27.2 & $0.64(0.39-1.06)$ & 0.08 & & & & & \\
\hline & $\begin{array}{l}12-22 \\
\text { repetitions }\end{array}$ & 100 & 38.0 & 1.00 & Referent & & & & & \\
\hline
\end{tabular}

Any injury (overuse and traumatic) 
Table 5 Univariate associations between injury risk ${ }^{a}$ and questionnaire variables

\begin{tabular}{|c|c|c|c|c|c|c|c|c|c|c|}
\hline \multirow{2}{*}{$\begin{array}{l}\text { Variable } \\
\text { Category }\end{array}$} & \multirow[t]{2}{*}{ Variable } & \multirow[t]{2}{*}{ Strata } & \multicolumn{4}{|c|}{ Men } & \multicolumn{4}{|c|}{ Women } \\
\hline & & & $\mathrm{N}$ & $\begin{array}{l}\text { Injured } \\
\text { (\%) }\end{array}$ & $\begin{array}{l}\text { Hazard Ratios } \\
(95 \% \mathrm{Cl}) \text { from } \\
\text { CoxRegressions }\end{array}$ & $\begin{array}{l}\text { p-value(from } \\
\text { WaldStatistic) }\end{array}$ & $\mathrm{N}$ & $\begin{array}{l}\text { Injured } \\
(\%)\end{array}$ & $\begin{array}{l}\text { Hazard Ratios } \\
(95 \% \mathrm{Cl}) \\
\text { fromCox } \\
\text { Regressions }\end{array}$ & $\begin{array}{l}\text { p-value(from } \\
\text { WaldStatistic) }\end{array}$ \\
\hline \multirow[t]{9}{*}{$\begin{array}{l}\text { Tobacco } \\
\text { Use }\end{array}$} & $\begin{array}{l}\text { Smoked } 100 \\
\text { Cigarettes in Lifetime }\end{array}$ & No & 352 & 36.9 & 1.00 & Referent & 90 & 41.1 & 1.00 & Referent \\
\hline & & Yes & 74 & 25.7 & $0.63(0.40-1.03)$ & 0.07 & 15 & 46.7 & $1.17(0.52-2.61)$ & 0.71 \\
\hline & Age Smoked First & NeverSmoked & 244 & 41.4 & 1.00 & Referent & 64 & 35.9 & 1.00 & Referent \\
\hline & & 6-17 years & 116 & 26.7 & $0.56(0.37-0.84)$ & $<0.01$ & 23 & 52.2 & $1.65(0.82-3.32)$ & 0.16 \\
\hline & & $\geq 18$ years & 66 & 25.8 & $0.56(0.34-0.94)$ & 0.03 & 18 & 50.0 & $1.39(0.65-3.01)$ & 0.40 \\
\hline & Smoked in Last 30 & No & 413 & 34.9 & 1.00 & Referent & 102 & 42.2 & 1.00 & Referent \\
\hline & & Yes & 13 & 38.5 & $1.04(0.42-2.56)$ & 0.92 & 3 & 33.3 & $0.69(0.09-4.97)$ & 0.71 \\
\hline & Smokeless Tobacco & No & 402 & 35.1 & 1.00 & Referent & 104 & 42.3 & 1.00 & Referent \\
\hline & & Yes & 24 & 33.3 & $0.92(0.45-1.88)$ & 0.82 & 1 & 0.0 & -—— & -—— \\
\hline \multirow{18}{*}{$\begin{array}{l}\text { Physical } \\
\text { Activity }\end{array}$} & Self Rating of Physical & Less Active & 6 & 83.3 & $4.60(1.88-11.26)$ & $<0.01$ & 3 & 66.7 & $2.18(0.53-9.06)$ & 0.28 \\
\hline & & $\begin{array}{l}\text { About the } \\
\text { same }\end{array}$ & 30 & 26.7 & $0.75(0.37-1.52)$ & 0.42 & 12 & 33.3 & $0.69(0.25-1.94)$ & 0.48 \\
\hline & & More Active & 390 & 34.9 & 1.00 & Referent & 90 & 42.2 & 1.00 & Referent \\
\hline & $\begin{array}{l}\text { Frequency of Aerobic } \\
\text { Exercise in Last } 2 \\
\text { Months }\end{array}$ & $\begin{array}{l}\leq 1 \text { time/ } \\
\text { week }\end{array}$ & 2 & 100.0 & $5.96(1.44-24.69)$ & 0.01 & 0 & - & -—— & -—- \\
\hline & & $\begin{array}{l}2-4 \text { times/ } \\
\text { week }\end{array}$ & 264 & 31.4 & $0.75(0.54-1.03)$ & 0.08 & 56 & 44.6 & $1.14(0.63-2.07)$ & 0.67 \\
\hline & & $\begin{array}{l}\geq 5 \text { times/ } \\
\text { week }\end{array}$ & 160 & 40.0 & 1.00 & Referent & 49 & 38.8 & 1.00 & Referent \\
\hline & $\begin{array}{l}\text { Duration of Aerobic } \\
\text { Exercise Last } 2 \text { Months }\end{array}$ & 0-30 minutes & 84 & 34.5 & $0.73(0.45-1.19)$ & 0.02 & 9 & 44.4 & $0.89(0.29-2.70)$ & 0.83 \\
\hline & & $\begin{array}{l}31-60 \\
\text { minutes }\end{array}$ & 257 & 32.3 & $0.70(0.48-1.03)$ & 0.07 & 68 & 38.2 & $0.77(0.40-1.45)$ & 0.40 \\
\hline & & $\geq 61$ minutes & 85 & 43.5 & 1.00 & Referent & 28 & 50.0 & 1.00 & Referent \\
\hline & $\begin{array}{l}\text { Frequency of Weight } \\
\text { Training in Last } 2 \\
\text { Months }\end{array}$ & $\begin{array}{l}\leq 1 \text { time/ } \\
\text { week }\end{array}$ & 104 & 40.4 & $1.40(0.78-2.52)$ & 0.26 & 24 & 41.7 & $0.90(0.25-3.28)$ & 0.88 \\
\hline & & $\begin{array}{l}\text { 2-4 times/ } \\
\text { week }\end{array}$ & 275 & 33.5 & $1.06(0.61-1.83)$ & 0.84 & 74 & 41.9 & $0.88(0.27-2.89)$ & 0.84 \\
\hline & & $\begin{array}{l}\geq 5 \text { times/ } \\
\text { week }\end{array}$ & 47 & 31.9 & 1.00 & Referent & 7 & 42.9 & 1.00 & Referent \\
\hline & Duration of Weight & 0-30 minutes & 123 & 33.3 & $0.80(0.49-1.31)$ & 0.38 & 42 & 40.5 & $0.46(0.19-1.10)$ & 0.08 \\
\hline & & $\begin{array}{l}31-60 \\
\text { minutes }\end{array}$ & 241 & 34.0 & $0.78(0.50-1.22)$ & 0.28 & 53 & 37.7 & $0.38(0.16-0.90)$ & 0.03 \\
\hline & & $\geq 61$ minutes & 62 & 41.9 & 1.00 & Referent & 10 & 70.0 & 1.00 & Referent \\
\hline & $\begin{array}{l}\text { Frequency of Playing } \\
\text { Sports Last } 2 \text { Months }\end{array}$ & $\begin{array}{l}\leq 1 \text { time/ } \\
\text { week }\end{array}$ & 376 & 35.4 & $0.61(0.09-4.36)$ & 0.62 & 91 & 40.7 & $0.76(0.34-1.71)$ & 0.51 \\
\hline & & $\begin{array}{l}2-4 \text { times/ } \\
\text { week }\end{array}$ & 48 & 31.3 & $0.50(0.07-3.82)$ & 0.51 & 14 & 50.0 & 1.00 & Referent \\
\hline & & $\begin{array}{l}\geq 5 \text { times/ } \\
\text { week }\end{array}$ & 2 & 50.0 & 1.00 & Referent & 0 & - & -—- & --- \\
\hline
\end{tabular}


Table 5 Univariate associations between injury risk ${ }^{\mathbf{a}}$ and questionnaire variables (Continued)

\begin{tabular}{|c|c|c|c|c|c|c|c|c|c|c|}
\hline & Duration of Sports in & 0-30 minutes & 253 & 36.8 & $1.01(0.64-1.59)$ & 0.97 & 59 & 42.4 & $0.85(0.37-1.97)$ & 0.71 \\
\hline & & $\begin{array}{l}31-60 \\
\text { minutes }\end{array}$ & 110 & 30.0 & $0.79(0.46-1.35)$ & 0.39 & 32 & 37.5 & $0.71(0.28-1.80)$ & 0.48 \\
\hline & & $\geq 61$ minutes & 63 & 36.5 & 1.00 & Referent & 14 & 50.0 & 1.00 & Referent \\
\hline \multirow{16}{*}{$\begin{array}{l}\text { Prior } \\
\text { Injury }\end{array}$} & Prior Lower Limb & No & 167 & 29.3 & 1.00 & Referent & 40 & 42.5 & 1.00 & Referent \\
\hline & & Yes & 259 & 38.6 & $1.45(1.03-2.04)$ & 0.03 & 65 & 41.5 & $1.07(0.59-1.97)$ & 0.82 \\
\hline & Lower Limb Injury & No Injury & 167 & 29.3 & 1.00 & Referent & 40 & 42.5 & 1.00 & Referent \\
\hline & & No & 41 & 34.1 & $1.17(0.65-2.12)$ & 0.62 & 10 & 30.0 & $0.73(0.22-2.50)$ & 0.62 \\
\hline & & Yes & 218 & 39.4 & $1.51(1.06-2.14)$ & 0.02 & 55 & 43.6 & $1.14(0.61-2.12)$ & 0.68 \\
\hline & Returned to Normal & No Injury & 168 & 29.8 & 1.00 & Referent & 40 & 42.5 & 1.00 & Referent \\
\hline & & No & 14 & 64.3 & $2.97(1.46-6.05)$ & $<0.01$ & 4 & 50.0 & $1.29(0.30-5.61)$ & 0.73 \\
\hline & & Yes & 244 & 36.9 & $1.35(0.95-1.91)$ & 0.09 & 61 & 41.0 & $1.06(0.57-1.96)$ & 0.86 \\
\hline & Injured Upper Limb & No & 248 & 31.0 & 1.00 & Referent & 73 & 41.1 & 1.00 & Referent \\
\hline & & Yes & 178 & 40.4 & $1.38(1.00-1.88)$ & 0.05 & 32 & 43.8 & $1.04(0.55-1.97)$ & 0.90 \\
\hline & Upper Limb Injury & No Injury & 248 & 31.0 & 1.00 & Referent & 73 & 41.1 & 1.00 & Referent \\
\hline & & No & 34 & 35.3 & $1.13(0.62-2.08)$ & 0.70 & 9 & 33.3 & $0.88(0.27-2.88)$ & 0.83 \\
\hline & & Yes & 144 & 41.7 & $1.43(1.02-2.00)$ & 0.04 & 23 & 47.8 & $1.10(0.55-2.20)$ & 0.79 \\
\hline & Returned to Normal & No Injury & 247 & 30.8 & 1.00 & Referent & 73 & 41.1 & $1.09(0.58-2.05)$ & 0.97 \\
\hline & & No & 11 & 72.7 & $2.93(1.41-6.08)$ & $<0.01$ & 1 & 0.0 & -—- & -—- \\
\hline & & Yes & 168 & 38.7 & 1.31 (0.94-1.83) & 0.11 & 31 & 45.2 & 1.00 & Referent \\
\hline \multirow{6}{*}{$\begin{array}{l}\text { Pain } \\
\text { Limiting } \\
\text { Activity }\end{array}$} & Have Foot Pain & No & 363 & 32.5 & 1.00 & Referent & 81 & 39.5 & 1.00 & Referent \\
\hline & & Yes & 63 & 492 & $1.64(1.10-2.44)$ & 0.01 & 24 & 50.0 & $1.46(0.75-2.84)$ & 0.27 \\
\hline & Have Knee Pain & No & 313 & 31.3 & 1.00 & Referent & 82 & 41.5 & 1.00 & Referent \\
\hline & & Yes & 113 & 45.1 & $1.67(1.19-2.34)$ & $<0.01$ & 23 & 43.5 & $1.24(0.61-2.51)$ & 0.55 \\
\hline & Have Back Pain & No & 341 & 32.8 & 1.00 & Referent & 84 & 35.7 & 1.00 & Referent \\
\hline & & Yes & 85 & 43.5 & $1.33(0.92-1.93)$ & 0.13 & 21 & 66.7 & $2.33(1.23-4.40)$ & $<0.01$ \\
\hline \multirow{7}{*}{$\begin{array}{l}\text { Menstrual } \\
\text { History }\end{array}$} & Age of Menarche & 8-11 Years & & & & & 9 & 22.2 & $0.43(0.10-1.77)$ & 0.24 \\
\hline & & 12-14 Years & & & & & 76 & 46.1 & 1.00 & Referent \\
\hline & & $\geq 15$ Years & & & & & 19 & 31.6 & $0.61(0.26-1.45)$ & 0.26 \\
\hline & Menstrual Periods Last & $1-10$ & & & & & 20 & 40.0 & $0.85(0.40-1.84)$ & 0.69 \\
\hline & & $11-13$ & & & & & 84 & 41.7 & 1.00 & Referent \\
\hline & Gone $\geq 6$ Months & No & & & & & 102 & 40.2 & 1.00 & Referent \\
\hline & & Yes & & & & & 2 & 100.0 & $2.84(0.69-11.77)$ & 0.15 \\
\hline
\end{tabular}


Table 5 Univariate associations between injury risk ${ }^{\mathbf{a}}$ and questionnaire variables (Continued)

\begin{tabular}{|c|c|c|c|c|c|}
\hline Used Birth Control in & No & 47 & 48.9 & $1.52(0.84-2.77)$ & 0.17 \\
\hline & Yes & 57 & 35.1 & 1.00 & Referent \\
\hline \multirow{2}{*}{$\begin{array}{l}\text { Used Hormonal } \\
\text { Therapy in Past } 12 \\
\text { Months }\end{array}$} & No & 97 & 41.2 & $0.88(0.27-2.85)$ & 0.83 \\
\hline & Yes & 7 & 42.9 & 1.00 & Referent \\
\hline \multirow[t]{2}{*}{ Ever Pregnancy } & No & 90 & 40.0 & 1.00 & Referent \\
\hline & Yes & 12 & 41.7 & $1.02(0.40-2.61)$ & 0.96 \\
\hline
\end{tabular}

${ }^{\mathrm{a}}$ Any injury (overuse and traumatic)

Table 6 Multivariate analysis of injury ${ }^{\mathrm{a}}$ risk factors

\begin{tabular}{|c|c|c|c|c|c|}
\hline Gender & Variable & Strata & $\mathbf{N}$ & $\begin{array}{l}\text { Hazard Ratios }(95 \% \mathrm{Cl}) \text { fromCox } \\
\text { Regressions }\end{array}$ & $\begin{array}{l}\text { p-value(from } \\
\text { Waldstatistic) }\end{array}$ \\
\hline \multirow[t]{23}{*}{ Men } & Age & 24.4-29.9 years & 187 & 1.00 & Referent \\
\hline & & 30.0-38.6 years & 238 & $1.70(1.20-2.42)$ & $<0.01$ \\
\hline & 300-Meter Sprint & 40-44 seconds & 122 & 1.00 & Referent \\
\hline & & 45-46 seconds & 132 & $1.34(0.84-2.16)$ & 0.22 \\
\hline & & $47-48$ seconds & 108 & $1.84(1.31-3.00)$ & 0.01 \\
\hline & & 49-55 seconds & 63 & $1.75(1.01-3.02)$ & 0.05 \\
\hline & 1.5-Mile Run & $\begin{array}{l}8.18-10.35 \\
\text { minutes }\end{array}$ & 108 & 1.00 & Referent \\
\hline & & $\begin{array}{l}10.36-11.10 \\
\text { minutes }\end{array}$ & 107 & $1.14(0.67-1.94)$ & 0.63 \\
\hline & & $\begin{array}{l}11.11-11.64 \\
\text { minutes }\end{array}$ & 104 & $1.84(1.11-3.04)$ & 0.02 \\
\hline & & $\begin{array}{l}11.65-15.02 \\
\text { minutes }\end{array}$ & 106 & $1.95(1.19-3.20)$ & $<0.01$ \\
\hline & Smoked 100 Cigarettesin Lifetime & No & 351 & 1.00 & Referent \\
\hline & & Yes & 74 & $0.42(0.25-0.71)$ & $<0.01$ \\
\hline & Self Rating of PhysicalActivity & Less Active & 6 & $6.68(2.52-17.70)$ & $<0.01$ \\
\hline & & About the Same & 30 & $0.56(0.27-1.17)$ & 0.12 \\
\hline & & More Active & 389 & 1.00 & Referent \\
\hline & Frequency of AerobicExercise in Last 2 Months & $\leq 1$ time/week & 2 & $4.58(0.97-21.71)$ & 0.06 \\
\hline & & 2-4 times/week & 263 & $0.69(0.49-0.97)$ & 0.03 \\
\hline & & $\geq 5$ times/week & 160 & 1.00 & Referent \\
\hline & Returned to NormalPhysical ActivitySince & No Injury & 246 & 1.00 & Referent \\
\hline & & No & 11 & $3.36(1.48-7.62)$ & $<0.01$ \\
\hline & & Yes & 168 & $1.41(1.00-2.00)$ & 0.05 \\
\hline & Have KneePain LimitingActivity Sometime & No & 313 & 1.00 & Referent \\
\hline & & Yes & 112 & $1.84(1.28-2.64)$ & $<0.01$ \\
\hline \multirow[t]{5}{*}{ Women } & 1.5-Mile Run & $\begin{array}{l}\text { 10.05-12.06 } \\
\text { minutes }\end{array}$ & 34 & 1.00 & Referent \\
\hline & & $\begin{array}{l}12.07-12.95 \\
\text { minutes }\end{array}$ & 32 & $1.00(0.46-2.18)$ & 0.99 \\
\hline & & $\begin{array}{l}\text { 12.96-14.92 } \\
\text { minutes }\end{array}$ & 32 & $1.95(0.97-3.93)$ & 0.06 \\
\hline & Have BackPain LimitingActivity Sometime & No & 78 & 1.00 & Referent \\
\hline & & Yes & 20 & $2.37(1.27-4.43)$ & $<0.01$ \\
\hline
\end{tabular}


training activity (e.g., handcuffing, breaking falls, boxing). Given the emphasis in the curriculum on defensive tactics and physical training and the physical demands of those activities, it is reasonable that they were responsible for most of the injuries. Observations of defensive tactics training revealed that a number of safety features were already in place. For example, during boxing new agents wore boxing gloves, headgear, and mouthpieces; during other defensive tactics training they practiced on cushioned mats, which offered some protection during falls and takedowns. Nonetheless, it is sensible that defensive tactics could be further examined to see if additional safety measures might be implemented.

Older male new agents were at significantly higher injury risk and older female new agents tended to be at higher risk. These findings are in consonance with those in military training [16-22] which also show that older individuals are at higher injury risk compared to younger individuals when they perform similar activities. The reason for the higher susceptibility to injury in older individuals may have to do with age-related changes in stem cells, declines in fitness, and/or prior injury history. The ability of resident stem cells to initiate and conduct tissue repair declines with age [23-25]. This could make older individuals more susceptible to overuse-type injuries, in which small microtraumas accumulate over time and repair in the older tissue does not keep pace with these repeated microtraumas. In the present investigation, only about $14 \%$ of injuries were of the overuse type, but it is possible that repetitive microtraumas, coupled with slower repair processes, may also weaken tissue to the point where sudden energy exchanges are more likely to cause acute (traumatic) tissue damage. With regard to fitness, aging results in a loss of muscle mass, muscle strength, muscular endurance, aerobic capacity, and flexibility [26,27]. The loss of aerobic capacity and muscular endurance can begin as early as age 25 [27]. These age-related changes reduce absolute fitness levels and may make injuries more likely, since lower fitness has been shown to be consistently related to injury $[15,17,18,28-39]$. Contrary to this hypothesis however, older age and lower aerobic fitness were found to be independent injury risk factors in the multivariate model. Further, there were low (non-significant) Pearson product-moment correlations between age and performance on all the fitness events (correlations ranged from -0.04 to 0.19 ). Thus, the lower potential fitness level of the older individuals is not likely to account for the relationship between age and injury. With regard to prior injuries, it is possible that older individuals may be more likely to have experienced prior injuries that may make them more susceptible to future injuries; prior injuries have been shown to be a risk factor for new injuries in many studies [34,40-50]. To examine this hypothesis, self-reported prior injuries were stratified by age, as shown in Table 7. Injuries in training were higher in the older men, regardless of whether or not they reported a prior upper or lower body injury. Results for the women were less clear, presumably because of the greater variability in the smaller sample. Nonetheless, injury-in-training risk still tended to be higher in the older women regardless of whether or not the women had a prior injury. In addition, older age and a prior upper limb injury that interfered with normal activity were both independent risk factors for training-related injuries in the multivariate analysis for men. Thus, the hypothesis that prior injury may make older individuals more susceptible to injuries in training was not supported in these data.

Lower levels of physical fitness were generally associated with higher injury risk, in agreement with our previous retrospective investigation [1]. In the present investigation, this was statistically significant only for the 300-meter run and the 1.5-mile run, but injury risk

Table 7 Injuries in training stratified by self-reported prior injury and age

\begin{tabular}{|c|c|c|c|c|c|c|c|c|}
\hline \multirow[t]{2}{*}{ Gender } & \multirow{2}{*}{$\begin{array}{l}\text { Self- } \\
\text { ReportedInjuryType }\end{array}$} & \multirow[t]{2}{*}{ ResponseCategory } & \multicolumn{2}{|c|}{ 24.1-29.9 Year Olds } & \multicolumn{2}{|c|}{ 30.0-38.6 Year Olds } & \multirow{2}{*}{$\begin{array}{l}\mathrm{p}- \\
\text { value }^{\mathrm{a}}\end{array}$} & \multirow{2}{*}{$\begin{array}{l}\text { Risk Ratio-Older/Younger } \\
(95 \% \mathrm{Cl})\end{array}$} \\
\hline & & & $\bar{N}$ & $\begin{array}{l}\text { \% Injuredin } \\
\text { Training }\end{array}$ & $\mathrm{N}$ & $\begin{array}{l}\text { \% Injuredin } \\
\text { Training }\end{array}$ & & \\
\hline \multirow[t]{4}{*}{ Men } & Lower Limblnjury & $\begin{array}{l}\text { No ReportedPrior } \\
\text { Injury }\end{array}$ & 73 & 21.9 & 94 & 35.1 & 0.06 & $1.60(0.96-2.67)$ \\
\hline & & ReportedPrior Injury & 115 & 29.6 & 144 & 45.8 & $<0.01$ & $1.55(1.11-2.16)$ \\
\hline & Upper Limblnjury & No Reportedlnjury & 113 & 23.9 & 135 & 37.0 & 0.03 & $1.55(1.04-2.30)$ \\
\hline & & ReportedInjury & 75 & 30.7 & 103 & 47.6 & 0.02 & $1.55(1.04-2.30)$ \\
\hline \multirow[t]{4}{*}{ Women } & Lower Limblnjury & No Reportedlnjury & 22 & 40.9 & 18 & 44.4 & 0.82 & $1.09(0.52-2.23)$ \\
\hline & & ReportedInjury & 38 & 34.2 & 27 & 51.9 & 0.15 & $1.52(0.86-2.68)$ \\
\hline & Upper Limblnjury & No Reportedlnjury & 45 & 37.8 & 28 & 46.4 & 0.47 & $1.23(0.71-2.12)$ \\
\hline & & Reportedlnjury & 15 & 33.3 & 17 & 52.9 & 0.27 & $1.59(0.68-3.69)$ \\
\hline
\end{tabular}

${ }^{\mathrm{a} C h i-s q u a r e ~ s t a t i s t i c ~}$ 
was still higher in the lower performing strata for all the fitness measures. In addition, slower 1.5-mile run time was an independent risk factor for injury in the multivariate analysis for both men and women. Of particular interest was the association between injuries and the total PFT point score. Both men and women who achieved at least 11-12 total PFT points were at lower injury risk than those who scored below 10 total points. Coincidentally, at least 12 points are currently required to "pass" the PFT at the FBI Academy.

The finding that FBI new agents with lower fitness levels were at higher risk of injury agrees well with military basic training studies $[15,17,18,28-39]$. However, this finding does not agree with most civilian studies [51-58] which generally find the opposite: individuals with higher fitness levels have higher injury incidence, presumably because higher fit individuals spend more time in physical activities that put them at risk. One of the common characteristics of military basic training and FBI new agent training is that individuals perform many physical activities with their fellow trainees. It is possible that the relationship between lower fitness and higher injury risk can be demonstrated in basic training and in new agent training because in these situations, the level and type of physical training are similar among participants. It is more difficult to demonstrate this association in civilian groups because of the greater variety in the levels and types of exercise and other physical activities.

Little association was found between current cigarette smoking and injury risk among the men or women in this investigation but it must be noted that that smoking frequency and amounts smoked were low in this group. Only 13 men and 3 women reported smoking one or more cigarette in the 30 days before new agent training. Also, the reported average amount of smoking was 2 and 6 cigarettes per day for the men and women, respectively. The combination of the small number of smokers and low cigarette dosage may account for the lack of a relationship. Many previous investigations [16-19,59-69] have shown that higher injury risk was associated with cigarette smoking, and that as the amount of smoking increases so does injury risk $[16-19,59,63]$.

The low prevalence of smoking in the present investigation contrasts with studies in the general population and studies of other law enforcement groups. The Centers for Disease Control and Prevention found that in 2008, 26\% of 22-44 year old men and 21\% of 22-44 year old women in the general US population reported smoking cigarettes. For those with undergraduate degrees, $12 \%$ of men and $10 \%$ of women reported smoking cigarettes. Studies of male law enforcement officers in various cities find smoking prevalence ranging from $12 \%$ to $51 \%$ [70-74].

In contrast to cigarette smoking, self-reported smokeless tobacco use among new FBI agents was similar to that of the general US population and Army recruits. Six percent of men $(n=24)$ and $1 \%$ of women $(n=1)$ reported using smokeless tobacco in the last 30 days. Data from the National Health Interview Survey indicated that in 2000, $6 \%$ of $25-44$ year old men and $0.3 \%$ of women of all ages use smokeless tobacco on all or most days [75]. In US Army Basic Combat Training in $1998,7 \%$ of men and 1\% of women reported smokeless tobacco use [31]. In the present prospective investigation, smokeless tobacco use was not associated with injury. This agrees with a previous studies in US Army Basic Combat Training, [31] but smokeless tobacco use has been found to increase injury risk in Norwegian basic training [17] and it is a risk factor for foot blisters $[76,77]$.

Somewhat perplexing was the lower injury risk among men who reported smoking at some point in their lives compared with those who had never smoked. Specifically, there was lower injury risk among men who reported smoking at least 100 cigarettes in their lives and/or those who reported smoking at some age. This is in contrast to studies of Army [78], Marine [79], and Air Force [36] recruits where there was higher injury risk among men who had smoked at least 100 cigarettes in their lives and/ or had smoked at some age. One difference in the FBI and military studies was that few FBI new agents were still smokers while a large proportion of military recruits (who are much younger than FBI new agents) continued to smoke up to the time they enter basic training. Many FBI new agents may have smoked in the past but did not continue this behavior. Beside smoking cessation, these individuals may have adopted other favorable health habits that may have reduced injury risk in training. It should also be noted that, contrary to the men in the present study, the women demonstrated a trend indicating lower injury risk among those who had smoked in the past.

Male new trainees who self-rated themselves as being less physically active than their peers, or who reported exercising $<=1$ times/week were at increased risk of injury compared to those in the highest activity categories. Women had a similar trend for self-rated physical activity, but no women reported performing aerobic exercise $\leq 1$ time/week. Although sample sizes were small in these less active categories, the findings are similar to that of Nabeel et al. [80] who reported that Minnesota police officers who were less physically active were more likely to report sprains, chronic pain, or back pain. The present data are also in consonance with previous studies of military training that found increased risk of injury among those who self-reported less physical activity relative to peers $[16,18,33,34,78,79,81]$, or a lower frequency of aerobic activity $[16,18,78,79]$. Physical activity of the proper intensity, frequency, and duration can increase 
aerobic fitness, muscle strength, and general health, and can reduce body fat [82-86]. Bone mineral density is higher in physically active individuals[60,87-89] and higher bone mineral density has been associated with greater weekly physical activity [88]. These and other factors may assist in reducing susceptibility to injury among more physically active individuals [90].

The American College of Sports Medicine (ACSM) recommends that, to promote and maintain health, 1865 year old men and women should perform moderately intense aerobic physical activity for at least 30 minutes on 5 days each week or vigorous activity for at least 20 minutes on 3 days each week [91,92]. To improve aerobic fitness $\left(\mathrm{VO}_{2} \mathrm{max}\right)$, long-term physical activity should be conducted at intensities between $50 \%$ and $90 \%$ of $\mathrm{VO}_{2} \max [93,94]$. Vigorous physical activity that promotes fitness would be most advantageous for FBI new agents because higher levels of fitness are associated with higher levels of occupational performance [95-97]. Data from the Behavioral Risk Factor Surveillance Study indicated that in 2009, 29\% of Americans reported that they performed the ACSM-recommended amount of vigorous activity [98]. ACSM also recommends that to promote and maintain good health, activities that increase muscular strength and muscular endurance should be performed on two or more days per week [91]. In the present investigation $95 \%$ of the men $(n=$ $405)$ and $96 \%$ of women $(n=101)$ reported vigorous aerobic exercise at least 3 times per week for at least 16-30 minutes; $78 \%$ of men $(n=332)$ and $89 \%$ of women $(n=93)$ reported vigorous aerobic exercise at least 3 times per week for at least 31-45 minutes. With regard to weight training, $78 \%$ of men $(n=332)$ and $77 \%$ of women $(n=81)$ reported weight training at least twice per week. Thus, a large proportion of new agents reported favorable amounts of physical activity in the 2 months before entering the FBI Academy.

Male FBI new agents who reported a prior upper or lower limb injury were at higher injury risk, especially if the injury prevented normal activity for 1 week, or if they were not able to eventually return to $100 \%$ of normal activity. Much of defensive tactics training involved upper body physical activity and those with upper body limitations may be more susceptible to injury during this training. Other studies of military groups $[41,42,47]$, athletes [43-46,48-50,99], and industrial workers [40] have reported an association between prior and current injuries, especially if the prior injury had occurred in the preceding year $[44,45,48-50]$. Many injuries may be chronic or recurrent, accounting for at least a part of this relationship. It should be noted that the multivariate analysis suggested a "graded" injury risk depending on the reported degree of recovery among those with a previous upper body injury. That is, individuals reporting a less than full recovery had a higher injury risk than those who do report a full recovery, or those reporting no previous injury at all; those who did report a full recovery were still at elevated risk compared to those who do not report a prior injury, but the magnitude of the risk was lower than those not reporting a full recovery.

Both men and women who self-reported foot, knee or back pain that limited activity tended to have elevated injury risk. This was statistically significant for foot and knee pain among the men and back pain among the women, but reported pain in any of these areas tended to increase injury risk. In addition, knee pain that limited activity among the men and back pain that limited activity among the women was independent risk factors for injury. It might be useful to screen prospective new agents who report a history of pain that limits activity.

\section{Limitations}

Injury diagnoses were limited to descriptions in the medical records and the CA-1 s. The medical database at the FBI Academy did not have standardized codes and diagnoses were determined from narrative descriptions. Many of these did not involve diagnostic tests that would have provided more definitive diagnoses. Nonetheless, the injury incidence, injury diagnoses, and anatomic locations of injuries were similar in both the prospective and retrospective investigations, which suggest the findings are reliable. These data provided a representative look at medical encounters and showed that the major types of injuries were strains, sprains, contusions and abrasions/lacerations, which are common injuries in physically active populations [6-16].

Data on tobacco use, physical activity, prior injury, and other questionnaire variables were based on selfreports, which could be subject to recall bias and social desirability bias. Nonetheless, data on physical activity and prior injury supports prior findings in military and other groups. Further, recall periods for many questions were limited to 30-60 days, which has been shown to improve the validity of questionnaire data [100-102]. The small number of tobacco users, although favorable from a health perspective, most likely limited the ability to find associations between tobacco use and injury, if indeed such a relationship exists among FBI new agents.

\section{Abbreviations}

FBI: Federal Bureau of Investigation; PFT: Physical Fitness Test; PFU: Physical Training Unit; PASW: Predictive Analytic Software; HR: Hazard Ratio; CDC: Centers for Disease Control and Prevention; ACSM: American College of Sports Medicine; 95\%Cl: 95\% Confidence Interval.

\section{Acknowledgements}

For their outstanding assistance with this investigation we would like to thank Ms Tanya Harvin, Special Agent Timothy Burke, Special Agent Gerald 
Jackson, Special Agent Susann Dreiling, Special Agent Jay Moeller, Special Agent Michael Vuogh, Dr. Tom Gross, Ms Terry Dotson, Ms Tammy Schuldt, Special Agent Melinda Casey, Dr David Wade, Ms Margaret Gray and Dr William Fabbri. This study was funded internally by the FBI and the US Army Public Health Command.

\section{Author details}

${ }^{1}$ U.S. Army Institute of Public Health, Aberdeen Proving Ground, Maryland, USA. ${ }^{2}$ Department of Health Policy and Management, Bloomberg School of Public Health, Johns Hopkins University, Baltimore, MD, USA. ${ }^{3}$ Federal Bureau of Investigation, Human Resources Division Office of Medical Services, Health Care Programs Unit, Washington, DC, USA.

\section{Authors' contributions}

JJK-Contributed to project design, data acquisition, data analysis, data interpretation, drafting and revising manuscript; TG-Contributed to data acquisition, data interpretation, revising manuscript; AS-Contributed to data acquisition, data analysis, data interpretation, drafting and revising manuscript; DS-Contributed to data acquisition, data analysis, data interpretation, revising manuscript; $\mathrm{KGH}$-Contributed to project design, data acquisition, data interpretation, revising manuscript; BG-Contributed to data acquisition, data interpretation, revising manuscript; JY-Contributed to project design, data interpretation, revising manuscript; $\mathrm{BHJ}-$ Contributed to project design, data interpretation, revising manuscript. All authors read and approved the final manuscript.

\section{Authors' Information}

The views, opinions, and/or findings contained in this report are those of the authors and should not be construed as official Federal Bureau of Investigation or Department of the Army position, policy or decision, unless so designated by other official documentation. Approved for public release; distribution is unlimited.

\section{Competing interests}

The authors declare that they have no competing interests.

Received: 12 May 2011 Accepted: 13 December 2011 Published: 13 December 2011

\section{References}

1. Knapik JJ, Spiess A, Swedler DI, Grier T, Hauret KG, Jones BH: Retrospective investigation of injury rates and physical fitness among Federal Bureau of Investigation new agent trainees, Quantico Virginia, 1999-2008. Aberdeen Proving Ground MD, US Army Public Health Command (Provisional), Technical Report No. 12-HF-97HRF1-09; 2010.

2. Federal Bureau of Investigation: FBI New Agent Physical Requirements., http://www.fbijobs.gov/1113.asp\#1 Accessed 18 July 2010.

3. Haddon W: On the escape of tigers: an ecological note. Am J Public Health 1970, 60:2229-2234.

4. National Institutes of Health: Clinical guidelines on the identification, evaluation, and treatment of overweight and obesity in adults. Bethesda MD, National Institutes of Health NIH Publication No. 98-4083; 1998.

5. Hosmer DW, Lemeshow S: Applied Logistic Regression New York: John Wiley \& Sons; 1989.

6. Brubaker CE, James SL: Injuries to runners. J Sports Med 1974, 2:189-198.

7. James SL, Bates BT, Osternig LR: Injuries to runners. Am J Sports Med 1978, 6:40-50.

8. McLain LG, Reynolds S: Sports injuries in a high school. Pediatrics 1989, 84:446-450.

9. Eisenberg I, Allen WC: Injuries in women's varsity athletic programs. Physician Sportsmed 1978, 6(3):112-120.

10. Knapik JJ, Bauman CL, Jones BH, Harris JM, Vaughan L: Preseason strength and flexibility imbalances associated with athletic injuries in female collegiate athletes. Am J Sports Med 1991, 19:76-81.

11. Jackson DS, Furman WK, Berson BL: Patterns of injuries in college athletes: a retrospective study of injuries sustained in intercollegiate athletics in two colleges over a two-year period. Mt Sinai J Med 1980, 47:423-426.

12. Lanese RR, Strauss RH, Leizman DJ, Rotondi AM: Injury and disability in matched men's and women's intercollegiate sports. Am J Public Health 1990, 80:1459-1462.
13. Watson AWS: Incidence and nature of sports injuries in Ireland: analysis of four types of sports. Am J Sports Med 1993, 21:137-143.

14. Maclntosh DL, Skrien T, Shephard RJ: Physical activity and injury. A study of sports injuries at the University of Toronto, 1951-1968. J Sports Med Phy Fitness 1972, 12:224-237.

15. Jones BH, Bovee MW, Harris JM, Cowan DN: Intrinsic risk factors for exercise-related injuries among male and female Army trainees. Am J Sports Med 1993, 21:705-710.

16. Jones BH, Cowan DN, Tomlinson JP, Robinson JR, Polly DW, Frykman PN: Epidemiology of injuries associated with physical training among young men in the Army. Med Sci Sports Exerc 1993, 25:197-203.

17. Heir T, Eide G: Injury proneness in infantry conscripts undergoing a physical training programme: smokeless tobacco use, higher age, and low levels of physical fitness are risk factors. Scand J Med Sci Sports 1997, 7:304-311.

18. Knapik JJ, Sharp MA, Canham-Chervak M, Hauret K, Patton JF, Jones BH: Risk factors for training-related injuries among men and women in Basic Combat Training. Med Sci Sports Exerc 2001, 33:946-954.

19. Knapik JJ, Swedler D, Grier T, Hauret KG, Bullock S, Williams K, Darakjy S, Lester $\mathrm{M}$, Tobler $\mathrm{S}$, Jones $\mathrm{BH}$ : Injury reduction effectiveness of selecting running shoes based on plantar shape. J Strength Cond Res 2009, 23:685-697.

20. Knapik JJ, Spiess A, Swedler D, Grier T, Darakjy S, Amoroso P, Jones BH: Injury risk factors in parachuting and acceptability of the parachute ankle brace. Aviat Space Environ Med 2008, 79:689-694.

21. Craig SC, Lee T: Attention to detail: injuries at altitude among U.S. Army military static line parachutists. Mil Med 2000, 165:268-271.

22. Schmidt MD, Sulski SI, Amoroso PJ: Effectiveness of an external ankle brace in reducing parachute-related ankle injuries. Med Sci Sports Exerc 2004, 36:S312

23. Schmitt R, Cantley LG: The impact of ageing on kidney repair. Am J Physiol Renal Physiol 2008, 294:F1265-F1272.

24. Conboy IM, Rando TA: Aging, stem cells and tissue regeneration. Cell Cycle 2005, 4:407-410.

25. Pittman J: Effects of aging on wound healing: current concepts. J Wound Ostomy Continence Nurs 2007, 34:412-415.

26. Barowclough F: The process of aging. J Adv Nur 1981, 6:319-325.

27. Knapik JJ, Jones BH, Vogel JA, Banderet LE, Bahrke MS, O'Connor JS: Influence of age and body mass index on measures of physical fitness in U.S. Army Soldiers. J Aging Phys Act 1996, 4:234-250.

28. Jones $\mathrm{BH}$, Bovee MW, Knapik JJ: Associations among body composition, physical fitness, and injuries in men and women Army trainees. In Body Composition and Physical Performance. Edited by: Marriott BM, GrumstrupScott J. Washington, D.C.: National Academy Press; 1992:141-173.

29. Westphal KA, Friedl KE, Sharp MA, King N, Kramer TR, Reynolds KL, Marchitelli LJ: Health, performance and nutritional status of U.S. Army women during basic combat training. Natick, MA, U.S. Army Research Institute of Environmental Medicine, Technical Report No. T96-2; 1995.

30. Knapik JJ, Cuthie J, Canham M, Hewitson W, Laurin MJ, Nee MA, Hoedebecke E, Hauret $\mathrm{K}$, Carroll D, Jones BH: Injury incidence, injury risk factors, and physical fitness of U.S. Army basic trainees at Ft Jackson SC, 1997. Aberdeen Proving Ground, MD, U.S. Army Center for Health Promotion and Preventive Medicine, Epidemiological Consultation Report No. 29-HE-7513-98; 1998.

31. Knapik JJ, Sharp MA, Canham ML, Hauret K, Cuthie J, Hewitson W, Hoedebecke E, Laurin MJ, Polyak C, Carroll D, et al: Injury incidence and injury risk factors among US Army Basic Trainees at Ft Jackson, SC (including fitness training unit personnel, discharges, and newstarts). Aberdeen Proving Ground MD, US Army Center for Health Promotion and Preventive Medicine, Epidemiological Consultation Report No. 29-HE-837099; 1999.

32. Almeida SA, Williams KM, Shaffer RA, Brodine SK: Epidemiological patterns of musculoskeletal injuries and physical training. Med Sci Sports Exerc 1999, 31:1176-1182.

33. Shaffer RA, Brodine SK, Almeida SA, Williams KM, Ronaghy S: Use of simple measures of physical activity to predict stress fractures in young men undergoing a rigorous physical training program. Am J Epidemiol 1999, 149:236-242

34. Rauh MJ, Macera CA, Trone DW, Shaffer RA, Brodine SK: Epidemiology of stress fracture and lower-extremity overuse injuries in female recruits. Med Sci Sports Exerc 2006, 38:1571-1577. 
35. Jones BH, Shaffer RA, Snedecor MR: Injuries treated in outpatient clinics: surveys and research data. Mil Med 1999, 164(Suppl 6):1-6-89.

36. Knapik JJ, Brosch LC, Venuto M, Swedler DI, Bullock SH, Gaines LS, Murphy RJ, Canada SE, Hoedebecke EL, Tobler SK, et al: Injury reduction effectiveness of prescribing running shoes based on foot shape in Air Force Basic Military Training. Aberdeen Proving Ground MD, Army Center for Health Promotion and Preventive Medicine, Technical Report No. 12MA-05SBA-08A; 2008

37. Bijur PE, Horodyski M, Egerton W, Kurzon M, Lifrak S, Friedman S: Comparison of injury during cadet basic training by gender. Arch Pediatr Adolesc Med 1997, 151:456-461.

38. Heir T, Eide G: Age, body composition, aerobic fitness and health condition as risk factors for musculoskeletal injuries in conscripts. Scand J Med Sci Sports 1996, 6:222-227.

39. Canham ML, McFerren MA, Jones BH: The association of injuries with physical fitness among men and women in gender integrated basic combat training units. MSMR 1996, 2:8-10, 12

40. Forde MS, Punnett L, Wegman DH: Prevalence of musculoskeletal symptoms in union ironworkers. J Occup Env Hyg 2005, 2:203-212.

41. Knapik JJ, McCollam R, Canham-Chervak M, Arnold S, DuVernoy TS, Hoedebecke EL: A second investigation of injuries among officers attending the US Army War College during Academic Year 2000. Aberdeen Proving Ground, MD, US Army Center for Health Promotion and Preventive Medicine, EPICON Report No. 29-HE-2682-00; 2000.

42. Knapik JJ, Spiess A, Darakjy S, Grier T, Manning F, Livingstone E, Swedler D, Amoroso $\mathrm{P}$, Jones BH: Risk factors for parachute injuries and comments about the parachute ankle brace among airborne students. Aberdeen Proving Ground MD, US Army Center for Health Promotion and Preventive Medicine, Technical Report No. 12-MA01Q2-08B; 2008.

43. Knowles SB, Marshall SW, Bowling JM, Loomis D, Millikan R, Yang J, Weaver NL, Kalsbeek W, Miller FO: A prospective study of injury incidence among North Carolina high school athletes. Am J Epidemiol 2006, 164:1209-1221

44. Macera CA, Pate RR, Powell KE, Jackson KL, Kendrick JS, Craven TE: Predicting lower-extremity injuries among habitual runners. Arch Int Med 1989, 149:2565-2568

45. Marti B, Vader JP, Minder CE, Abelin T: On the epidemiology of running injuries. The 1984 Bern Grand-Prix study. Am J Sports Med 1988 16:285-294.

46. Orchard JW: Intrinsic and extrinsic risk factors for muscle strains in Australian football. Am J Sports Med 2001, 29:300-303.

47. Schneider GA, Bigelow C, Amoroso PJ: Evaluating risk of re-injury among 1,214 Army Airborne soldiers using a stratified survival model. Am J Prev Med 2000, 18(Suppl3):156-163.

48. VanMechelen W, Twisk J, Molendijk A, Blom B, Snel J, Kemper HC: Subjectrelated risk factors for sports injuries: a 1-yr prospective study in young adults. Med Sci Sports Exerc 1996, 28:1171-1179.

49. Voaklander DC, Saunders LD, Quinney HA: Personal risk factors for injury in recreational and old-timer ice hockey. Sports Med Training Rehabil 1998, 8:239-250

50. Walter SD, Hart LE, Mclntosh JM, Sutton JR: The Ontario cohort study of running-related injuries. Arch Int Med 1989, 149:2561-2564

51. Blair SN, Kohl HW, Goodyear NN: Rates and risks for running and exercise injuries: studies in three populations. Res Quart Exerc Sports 1987 58:221-228.

52. Hootman JM, Macera CA, Ainsworth BE, Martin M, Blair SN: Predictors of lower extremity injury among recreationally active adults. Clin J Sport Med 2002, 12:99-106.

53. Hootman JM, Macera CA, Ainsworth BE, Addy CL, Martin M, Blair SN: Epidemiology of musculoskeletal injuries among sedentary and physically active adults. Med Sci Sports Exerc 2002, 34:838-844.

54. Hootman JM, Macera CA, Ainsworth BE, Martin M, Addy CL, Blair SN: Association among physical activity level, cardiorespiratory fitness, and risk of musculoskeletal injury. Am J Epidemiol 2001, 154:251-258.

55. Colbert LH, Hootman JM, Macera CA: Physical activity-related injuries in walkers and runners in the Aerobics Center Longitudinal Study. Clin J Sport Med 2000, 10:259-263.

56. Macera CA, Jackson KL, Hagenmaier GW, Kronenfeld JJ, Kohl HW, Blair SN: Age, physical activity, physical fitness, body composition and incidence of orthopeadic problems. Res Quart Exerc Sports 1989, 60:225-233.
57. Verstappen FTJ, Twellaar M, Hartgens F, vanMechelen W: Physical fitness and sports skills in relation to sports injuries. A four year prospective investigation of sports injuries among physical education students. Int J Sports Med 1998, 19:586-591.

58. Ready AE, Boreskie SL, Law SA, Russell R: Fitness and lifestyle parameters fail to predict back injuries in nurses. Can J Appl Physiol 1993, 18:80-90

59. Altarac M, Gardner JW, Popovich RM, Potter R, Knapik JJ, Jones BH: Cigarette smoking and exercise-related injuries among young men and women. Am J Prev Med 2000, 18(Suppl 3S):96-102.

60. Lappe JM, Stegman MR, Recker RR: The impact of lifestyle factors on stress fractures in female Army recruits. Osteoporosis Int 2001, 12:35-42.

61. Valimaki W, Alfthan E, Lehmuskallio E, Loyttyniemi E, Sah T, Suominen H, Valimakii MJ: Risk factors for clinical stress fractures in male military recruits: a prospective cohort study. Bone 2005, 37:267-273.

62. Reynolds KL, Heckel HA, Witt CE, Martin JW, Pollard JA, Knapik JJ, Jones BH: Cigarette smoking, physical fitness, and injuries in infantry soldiers. Am J Prev Med 1994, 10:145-150.

63. Knapik JJ, Brosch LC, Venuto M, Swedler DI, Bullock SH, Gaines LS, Murphy RJ, Tchandja J, Jones BH: Effect on injuries of assigning shoes based on foot shape in Air Force Basic Training. Am J Prev Med 2010, 38 : S197-S211.

64. Cady LD, Bischoff DP, O'Connell ER, Thomas PC, Allen JH: Strength and fitness and subsequent back injuries in firefighters. J Occ Med 1979, 21:269-272.

65. Chau N, Bourgkard E, Bhattacherjee A, Ravaud JF, Choquet M, Mur JM: Associations of job, living conditions and lifestyle with occupational injury in working population: a population-based study. Int Arch Occup Environ Health 2008, 81:379-389.

66. McSweeney KP, Congleton JJ, Kerk CJ, Jenkins OE, Craig BN: Correlation of recorded injury and illness data with smoking, exercise, and absolute aerobic capacity. Int J Ind Ergonomics 1999, 24:193-200.

67. Craig BN, Congleton JJ, Kerk CJ, Amendola AA, Gaines WG: Personal and non-occupational risk factors and occupational injury/illness. Am J Ind Med 2006, 49:249-260.

68. Mudr D, Naus A, Hetychova V, Vavreckova O: Work injuries and smoking. Ind Med Surg 1966, 35:880-881.

69. Ryan J, Zwerling C, Orav EJ: Occupational risk associated with cigarette smoking: a prospective study. Am J Public Health 1992, 82:29-32.

70. Sparrow D, Thomas HE, Weiss ST: Coronary artery disease in police officers participating in the Normative Aging Study. Am J Epidemiol 1983, 118:508-513.

71. Young DR, Steinhardt MA: The importance of physical fitness versus physical activity for coronary artery disease risk factors: a cross-sectional analysis. Res Quart Exerc, Sports 1993, 64:377-384.

72. Franke WD, Ramey SL, Shelley MC: Relationship between cardiovascular disease morbidity, risk factors, and stress in a law enforcement cohort. J Occ Environ Med 2002, 44:1182-1188.

73. Ramey SL: Cardiovascular disease risk factors and perception of general health among law enforcement officers. AAOHNJ 2003, 51:219-226.

74. Williams MA, Petratis MM, Baechle TR, Ryschon KL, Campain JJ, Sketch MH: Frequency of physical activity, exercise capacity, and atherosclerotic heart disease risk factors in male police officers. J Occ Med 1987, 29:596-600.

75. Nelson DE, Mowery P, Tomar S, Marcus S, Giovino G, Zhao L: Trends in smokeless tobacco use among adults and adolescents in the United States. Am J Public Health 2006, 96:897-905.

76. Knapik JJ, Reynolds K, Barson J: Risk factors for foot blisters during road marching: tobacco use, ethnicity, foot type, previous illness and other factors. Mil Med 1999, 164:92-97.

77. Reynolds K, Williams J, Miller C, Mathis A, Dettori J: Injuries and risk factors in an 18-day Marine winter mountain training exercise. Mil Med 2000, 165:905-910

78. Knapik JJ, Swedler D, Grier T, Hauret KG, Bullock S, Williams K, Darakjy S, Lester M, Tobler S, Clemmons N, et al: Injury reduction effectiveness of prescribing running shoes based on foot shape in basic combat training. Aberdeen Proving Ground MD, US Army Center for Health Promotion and Preventive Medicine, Technical Report No. 12-MA-05SB-08; 2008

79. Knapik JJ, Trone D, Swedler DI, Villasenor A, Schmied E, Bullock S, Jones BH: Injury reduction effectiveness of assigning running shoes based on foot shape in Marine Corps basic training. Aberdeen Proving Ground MD, US 
Army Center for Health Promotion and Preventive Medicine, No. 12-MA05SBA-08B; 2009

80. Nabeel I, Baker BA, McGrail MP, Flottemesch TJ: Correlation between physical activity, fitness, and musculoskeletal injuries in police officers. Minn Med 2007, 90:40-43.

81. Gardner LI, Dziados JE, Jones BH, Brundage JF, Harris JM, Sullivan R, Gill P: Prevention of lower extremity stress fractures: a controlled trial of a shock absorbent insole. Am J Public Health 1988, 78:1563-1567.

82. ACSM: The recommended quantity and quality of exercise for developing and maintaining cardiorespiratory and muscular fitness, and flexibility in healthy adults. Med Sci Sports Exerc 1998, 30:975-991.

83. Blair SN, Cheng Y, Holder JS: Is physical activity or physical fitness more important in defining health benefits? Med Sci Sports Exerc 2001, 33: S379-S399.

84. Kell RT, Bell G, Quinney A: Musculoskeletal fitness, health outcomes and quality of life. Sports Med 2001, 31:863-873.

85. Kohrt WM, Bloomfield SA, Little KD, Nelson ME, Yingling VR: Physical activity and bone health. Position stand of the American College of Sports Medicine. Med Sci Sports Exerc 2004, 36:1985-1996.

86. Ross R, Janssen I: Physical activity, total and regional obesity: doseresponse considerations. Med Sci Sports Exerc 2001, 33:S521-S527.

87. Afghani A, Xie B, Wiswell RA, Gong J, Li Y, Johnson CA: Bone mass of Asian adolescents in China: influence of physical activity and smoking. Med Sci Sports Exerc 2003, 35:720-729.

88. Babaroutsi E, Magkos F, Manios Y, Sidossis LS: Lifestyle factors affecting heel ultrasound in Greek females across different life stages. Osteoporosis Int 2005, 16:552-561.

89. Valimaki MJ, Karkkainen M, Lamberg-Allardt C, Laitinen K, Alhava E, Heikkinen J, Impivaara O, Makela P, Palmgren J, Seppanen R: Exercise, smoking, and calcium intake during adolescence and early adulthood as determinants of peak bone mass. Cardiovascular Risk in Young Finns Study Group. Br Med J 1994, 309:230-235.

90. Knapik JJ, Darakjy S, Hauret KG, Canada S, Scott S, Rieger W, Marin R, Jones $\mathrm{BH}$ : Increasing the physical fitness of low fit recruits before Basic Combat Training: an evaluation of fitness, injuries and training outcomes. Mil Med 2006, 171:45-54.

91. Haskell WL, Lee IM, Pate RR, Powell KE, Blair SN, Franklin BA, Macera CA, Heath GW, Thompson PD, Bauman A: Physical activity and public health: updated recommendations for adults from the American College of Sports Medicine and American Heart Association. Med Sci Sports Exerc 2007, 39:1423-1434.

92. American College of Sports Medicine: The recommended quantity and quality of exercise for developing and maintaining cardiorespiratory and muscular fitness in healthy adults. Med Sci Sports Exerc 1990, 22:265-274.

93. Wenger HA, Bell GJ: The interactions of intensity, frequency and duration of exercise training in altering cardiorespiratory fitness. Sports Med 1986, 3:346-356.

94. Gormley SE, Swain DP, High R, Spina RJ, Dowling EA, Kotipalli US, Gandrakota R: Effect of intensity of aerobic training on $\mathrm{VO}_{2}$ max. Med SCi Sports Exerc 2008, 40:1336-1343.

95. Sharp MA: Physical fitness and occupational performance of women in the U.S. Army. Work 1994, 4:80-92.

96. Knapik JJ, Daniels W, Murphy M, Fitzgerald P, Drews F, Vogel J: Physiological factors in infantry operations. Eur J Appl Physiol Occ Physiol 1990, 60:233-238.

97. Knapik JJ: The influence of physical fitness training on the manual material handling capability of women. Appl Ergonomics 1997, 28:339-345.

98. Prevalence and Trend Data. Physical Activity. 2009, http://apps.nccd.cdc gov/BRFSS/list.asp?cat $=$ PA\&yr $=2009 \& q k e y=4419 \&$ state $=$ All,.Accessed 11 August 2011.

99. Rauh MJ, Koepsell TD, Rivara FP, Margherita AJ, Rice SG: Epidemiology of musculoskeletal injuries among high school cross-country runners. Am J Epidemiol 2006, 163:151-159.

100. Zwerling C, Sprince NL, Wallace RB, Davis CS, Whitten PS, Heeringa SG: Effect of recall period on the reporting of occupational injuries among older workers in the Health and Retirement Study. Am J Ind Med 1995, 28:583-590.

101. Landen DD, Hendricks S: Effect of recall on reporting of at-work injuries. Public Health Rep 1995, 110:350-354.
102. Massey JT, Gonzalez JF: Optimal recall period for estimating accidental injuries in the National Health Interview Survey. Proc Am Stat Assoc 1976, 18:584-588.

\section{Pre-publication history}

The pre-publication history for this paper can be accessed here:

http://www.biomedcentral.com/1471-2458/11/920/prepub

doi:10.1186/1471-2458-11-920

Cite this article as: Knapik et al:: Injury rates and injury risk factors among Federal Bureau of Investigation new agent trainees. BMC Public Health 2011 11:920.

\section{Submit your next manuscript to BioMed Central and take full advantage of:}

- Convenient online submission

- Thorough peer review

- No space constraints or color figure charges

- Immediate publication on acceptance

- Inclusion in PubMed, CAS, Scopus and Google Scholar

- Research which is freely available for redistribution

Submit your manuscript at www.biomedcentral.com/submit
Ciomed Central 